\title{
An Inflammatory Reaction to Stored Fascia Lata 37 Years Postimplantation
}

\author{
Kaisra Esmail ${ }^{\mathrm{a}}$ David Ronald Jordan ${ }^{\mathrm{a}}$ Seymour Brownstein ${ }^{\mathrm{a}, \mathrm{b}}$ Tina Tang ${ }^{\mathrm{a}, \mathrm{b}}$ \\ Bruce Burns $^{\text {b }}$

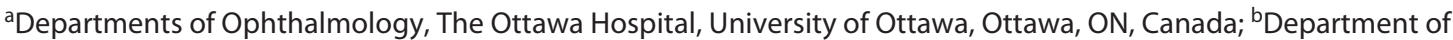 \\ Pathology, The Ottawa Hospital, University of Ottawa, Ottawa, ON, Canada
}

\section{Established Facts}

- Stored donor fascia lata has been used in ophthalmology as a suspensory material in frontalis sling ptosis surgery for decades with minimal complications and has occasionally been associated with early onset of localized inflammatory reactions, infection, and granuloma formation.

\section{Novel Insights}

- We report a rare suspected delayed localized granulomatous inflammatory reaction to stored irradiated fascia lata 37 years post-bilateral congenital ptosis surgery raising a concern whether donor tissue can ever truly be risk free.

\section{Keywords}

Donor fascia lata $\cdot$ Facia lata $\cdot$ Frontalis sling $\cdot$ Congenital ptosis

\footnotetext{
Abstract

We report a rare case of a suspected inflammatory reaction to stored fascia lata 37 years post-placement. Clinical, imaging, histopathological, and immunohistochemical findings are presented, with a literature review on reactions to stored fascia lata. A 39-year-old woman had upper eyelid congenital ptosis repaired successfully at 2 years with bilateral frontalis suspension procedures using stored fascia lata. Thirtyseven years later, the patient presented with swelling of her eyelids and forehead, which was tender to the touch, in the same pattern as the fascia lata slings placed earlier. Histopathological examination disclosed a non-necrotizing gran-
}

ulomatous inflammatory infiltrate with numerous asteroid bodies. Initially, it was responsive to oral prednisone, but with recurrent inflammation, long-term methotrexate was required to control the inflammation. To our knowledge, this type of delayed inflammatory reaction has not been previously reported. It raises a concern about the use of allogeneic donor tissue and accepted sterilization techniques that may not be $100 \%$ effective in deactivating all components of the donor graft, including potential infectious pathogens, leading to a subsequent latent reaction.

(c) 2021 S. Karger AG, Basel

Presented in part at the Annual Meeting of the Canadian Ophthalmological Society, Metro Toronto Convention Center, Toronto, ON, May 31-June 3, 2018, and The Verhoeff-Zimmerman Society Annual Meeting, Duke University, Durham, NC, April 3-7, 2019.

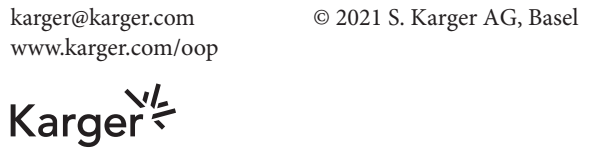

David Ronald Jordan

University of Ottawa Eye Institute, Ophthalmology

501 Smythe Rd.

Ottawa K1H 8L6 (Canada)

jordan1897@ rogers.com 


\section{Introduction}

Stored fascia lata has been used in several surgical specialties including ophthalmology, neurosurgery, urogynecology, oral surgery, and cosmetic surgery. Within ophthalmology, it has been utilized as a suspensory material in frontalis sling surgery, as an orbital floor implant, and as a patch graft during glaucoma filtering surgery [1-3].

Fascia lata for frontalis suspension surgery has been in use for over 100 years, provides excellent long-term results, and is considered the gold standard material for congenital ptosis when a frontalis sling is required [1, 4-7]. In children less than 3 years, there may be insufficient autologous iliotibial fascia lata available for harvest. Stored fascia lata has been utilized as an alternative as well as a variety of synthetic allografts (e.g., silicone rods, polypropylene, polyfilament nylon, polyester mesh, and polytetrafluoroethylene) [1,5-8]. Crawford $[7,9]$ pioneered the use of stored fascia lata in 1956 and preferred it to the alternative materials [1]. Ocular complications were uncommon but included early localized inflammatory reactions, infection, and granuloma formation $[1,4-6]$. We report a suspected delayed inflammatory reaction to stored irradiated fascia lata 37-year post-ptosis surgery and discuss whether it may be a latent reaction to a component of the allogeneic graft that was not completely inactivated by the original sterilization technique.

\section{Case Report}

A 39-year-old woman had upper eyelid congenital ptosis repaired successfully at 2 years with bilateral frontalis suspension procedures (by JS Crawford, University of Toronto) using stored fascia lata. The stored fascia lata was reported as having been placed bilaterally in a pentagonal fashion and that the surgery was uncomplicated and the postoperative recovery was uneventful.
Thirty-seven years later, the patient presented with eyelid and forehead swelling, which was tender to the touch, in the same pattern as the fascia lata slings placed earlier (Fig. 1a). An incisional biopsy was carried out on one of the swollen areas above the left brow.

Histopathologic examination disclosed a non-necrotizing granulomatous inflammatory infiltrate of the soft tissues of the superotemporal left forehead. Distinct "naked tubercles" (Fig. 2a) contained numerous multinucleated giant cells with various amounts of intracytoplasmic vacuoles and several asteroid bodies (Fig. 2b-d). The granulomas were mostly nonconfluent with a mild nongranulomatous infiltrate, predominantly lymphocytes, in the intervening connective tissue. CD163 was diffusely positive throughout the granulomas (Fig. 2d). The specimen stained negative with infectious stains, including the Hucker-Conn Gram, acid-fast, and Grocott's methenamine silver. Under polarized light, native collagen birefringence was noted around the granulomas. The Congo red stain was negative with no apple-green birefringence.

The patient's current and past medical history included wellcontrolled hypothyroidism. There was no other medical illness. A chest X-ray, blood work as part of an internal medicine consultation looking for other granulomatous diseases, including sarcoidosis, was negative.

The patient's forehead regions initially responded to a 10 -week course of oral prednisone $(70 \mathrm{mg} /$ day, tapering by $5 \mathrm{mg}$ every 5 days), and the inflammatory reaction disappeared completely. It recurred 10 weeks later in the same areas. The patient was reluctant to restarting oral prednisone due to the psychological effects of her first course but agreed to local triamcinolone injections. The forehead lesions were injected at 6 sites above each brow with $0.2 \mathrm{~cm}^{3}$ of triamcinolone $\left(40 \mathrm{mg} / \mathrm{cm}^{3}\right)$. A partial response occurred with decreased inflammation. With persistence of the inflammation and reluctance to going back on oral steroids, oral methotrexate was initiated with a $25-\mathrm{mg}$ tapering dose of prednisone over 4 weeks. Over the next 8 weeks, the inflammatory lesions resolved completely. There were no additional flare-ups until 2 years later when the patient was asked to stop the methotrexate prior to dental surgery. The inflammatory forehead lesions began to recur within 4 weeks of discontinuing the methotrexate. They again resolved once the methotrexate was restarted. She has remained on methotrexate $20 \mathrm{mg}$ per week and has been followed for an additional 2 years with no further flare-ups and no other medical illness (Fig. 1b).
Fig. 1. a Initial presentation of eyelid and forehead lumpy swelling in a similar pattern as the original fascia lata slings. b Resolution of swelling following post management on methotrexate. Mild residual ptosis persisted in the left upper eyelid.
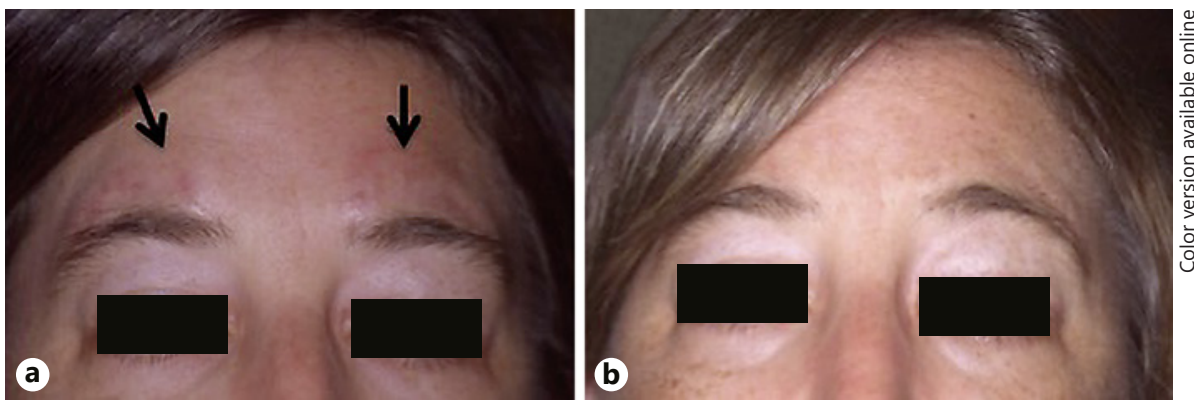
Fig. 2. a Distinct "naked tubercles" with numerous multinucleated giant cells are mostly nonconfluent surrounded by nongranulomatous infiltrate, predominantly lymphocytes, in the intervening connective tissue (hematoxylin and eosin stain, $\times 200$ ). b Multinucleated giant cell with various amounts of vacuoles and asteroid bodies (black boxes) (hematoxylin and eosin stain, $\times 200)$. c Numerous multinucleated giant cells with various amounts of vacuoles with some containing asteroid bodies (black boxes) (hematoxylin and eosin stain, $\times 200)$. d Immunohistochemical positive staining for macrophages with CD163 diffusely throughout the granulomas (hematoxylin and eosin stain, $\times 400$ ).
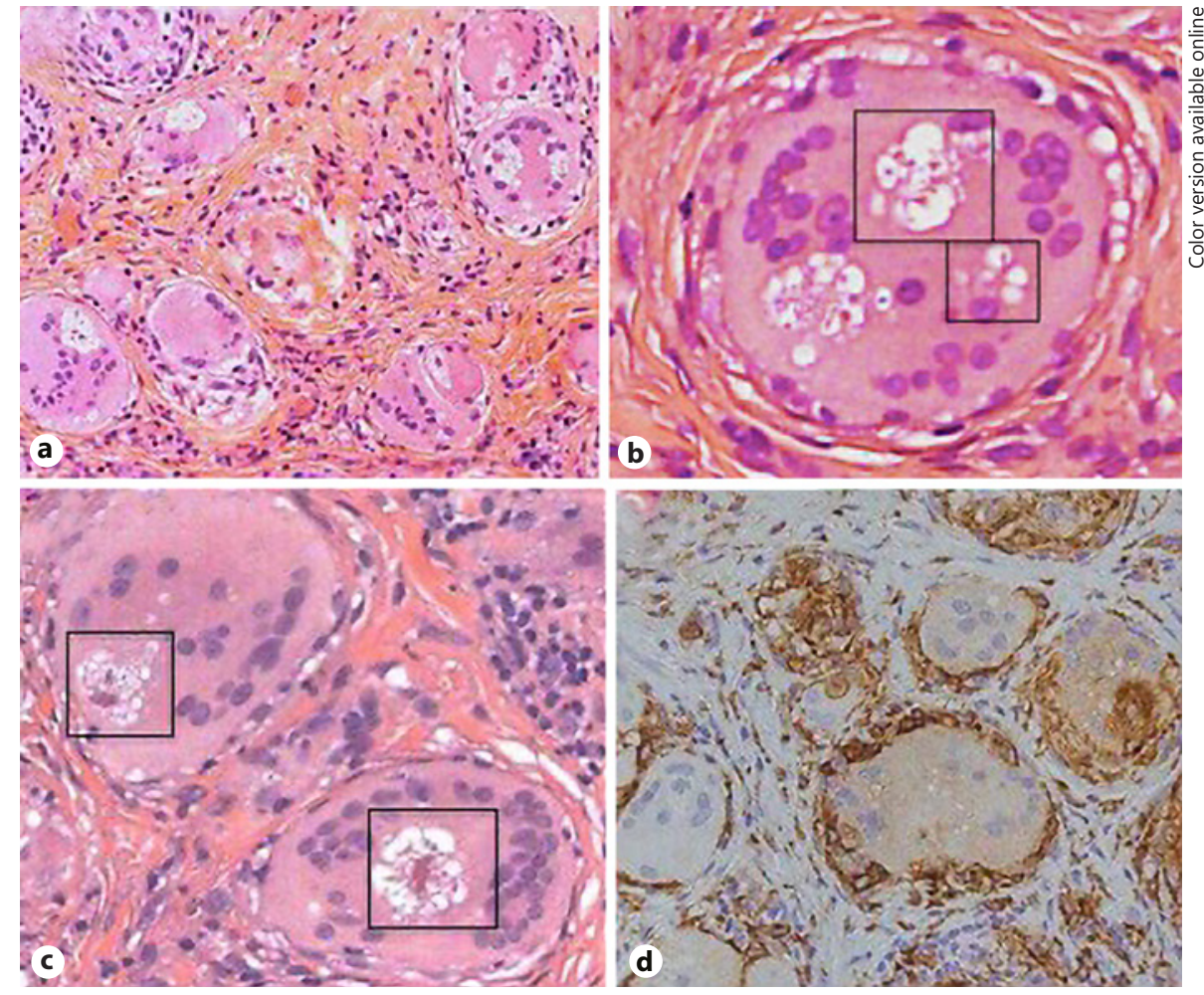

\section{Discussion}

Stored fascia lata has worked well as a satisfactory sling material for ptosis surgery over the past several decades [1, 4-7, 9-14]. Crawford reported complications over a 20 -year time period and described early brow inflammatory reactions and ptosis recurrence in some individuals [7]. Others have also described this inflammatory brow reaction in the immediate postoperative period with sterile abscess formation, as well as granuloma formation [4, $9,14,15]$. However, all of these reactions have been selflimited, occurred early in the postoperative period, and resolved without intervention. Stored fascia lata removed from postmortem patients 16 and 42 years postimplantation on microscopic exam suggested its permanent integration into surrounding structures with no signs of significant inflammation or reabsorption $[11,16]$.

In our present case, as sarcoidosis has been ruled out by an extensive systemic and ocular workup, as well as in part by the histopathological findings, the presence of the granulomas of the forehead could be due to a foreign body reaction. Furthermore, the cytoplasmic vesicles in the granulomas suggest that the granulomas responded to the foreign nature of the allogeneic fascia lata. In addition, the finding of native collagen surrounding most of the individual granulomas further supports that the diagnosis is not sarcoidosis.

The stored fascia lata in the present case was obtained from the University of Toronto Eye Bank in 1972. Preservation methods were based on accepted methods originally described in 1962 [1, 11, 17, 18]. Donors were between 20 and 50 years and did not die of protracted illness, neoplasm, septicemia, or viral illness. The fascia lata was harvested under sterile conditions and subjected to 3-4 million rads of gamma radiation from a Cobalt -60 source $[1,11,17,18]$.

Any donor material carries a theoretical risk of disease transmission and requires appropriate processing to remove this risk [4]. Sterilization techniques and standards have changed dramatically since 1962 and tissue screening standards, preparation, and processing continue to become more rigorous. Although safeguards are employed to ensure the safety of donor homologous tissue, it is important to determine whether donor tissue can ever truly be free of risk. The prevalence of diseases such as HIV, Hepatitis B and C, and the emerging concern surrounding prion diseases have led to a reluctance among some patients to accept any form of donor material that is not absolutely necessary $[4,19]$. 
Preservation techniques in the past and perhaps those currently being used in some centers may not be inactivating all components of donor tissue that may potentially induce a reaction and/or disease process even decades later, as illustrated with prion diseases [19]. Although we have no evidence prions were even remotely contributing to our patient's inflammatory reaction, we use it as an example to illustrate how difficult it can be to completely sterilize and/or neutralize donor tissue [19-24].

In summary, banked irradiated fascia lata has been used for decades as a frontalis sling material in congenital ptosis with poor levator function. It is well tolerated and has a low complication rate; the only documented reactions to date have occurred in the early postoperative period and were self-limited in nature. The unusual inflammatory reaction in the patient described herein is distinctly rare, has an unknown etiology, and to our knowledge, has not been previously reported. We suspect it may be a delayed inflammatory reaction to a component of the allogeneic fascia lata graft that was not completely inactivated by the original sterilization technique.

\section{Statement of Ethics}

This research was conducted ethically in accordance with the World Medical Association Declaration of Helsinki. The subject in the paper has given written informed consent to publish the case including publication of their images. Information revealing the subject's identity has been avoided.

\section{Conflict of Interest Statement}

The authors have no conflicts of interest to declare.

\section{Funding Sources}

There was no financial support for this case report.

\section{Author Contributions}

K. Esmail (Ophthalmology resident) pulled over 30 reference articles, summarized them, and provided the senior author (D.R.J.) with a brief synopsis of each. Dr. Esmail made a substantial contribution with respect to acquisition and analysis of previous work. She also presented the paper at the Canadian Ophthalmological society meeting. I estimate her work at $35 \%$ of the paper. D. Jordan drafted and fine-tuned the case report including abstract, case report, and discussion; I estimate his work at $35 \%$ of the paper. T. Tang was the pathology fellow for Dr. S. Brownstein and Dr. B. Burns. I estimate the pathology department's contribution to the paper as $30 \%$. Dr. T. Tang pulled additional reference articles and summarized them from the pathology standpoint. Dr. Brownstein and Dr. Burns provided the appropriate histopathologic specimens and made sure their description was accurate in the text. Dr. Brownstein presented the paper at the Verhoeff-Zimmerman Society meeting. All individuals in the paper made substantial contributions to the design of the work, acquisition and analysis, and final interpretation (review) of the paper.

\section{References}

1 Esmaeli B, Chung H, Pashby RC. Long-term results of frontalis suspension using irradiated, banked fascia lata. Ophthal Plast Reconstr Surg. 1998;14(3):159-63.

2 Bedrossian EH. HIV and banked fascia lata. Ophthal Plast Reconstr Surg. 1991;7(4): 284-8.

3 Tanji TM, Lundy DC, Minckler DS, Heuer DK, Varma R. Fascia lata patch graft in glaucoma tube surgery. Ophthalmology. 1996; 103(8):1309-12.

4 O’Reilly J, Lanigan B, Bowell R, O'Keefe M. Congenital ptosis: long-term results using stored fascia lata. Acta Ophthalmol Scand. 1998;76:346-8.

5 Wasserman BN, Sprunger DT, Helveston EM. Comparison of materials used in frontalis suspension. Arch Ophthalmol. 2001; 119(5):687-91.
6 Hersh D, Martin FJ, Rowe N. Comparison of silastic and banked fascia lata in pediatric frontalis suspension. J Pediatr Ophthalmol Strabismus. 2006;43(4):212-8.

7 Crawford JS. Repair of ptosis using frontalis muscle and fascia lata: a 20-year review. Ophthalmic Surg. 1977;8(4):31-40.

8 Ahmadi AJ, Sires BS. Ptosis in infants and children. Int Ophthalmol Clin. 2002;42(2): 15-29.

9 Crawford JS. Frontalis sling operation. J Ped Ophthalmol Stabismus. 1982;19(5):253-5.

10 Wagner RS, Mauriello JA, Nelson LB, Calhoun JH, Flanagan JC, Harley RD. Treatment of congenital ptosis with frontalis suspension: a comparison of suspensory materials. Ophthalmology. 1984;91(3):245-8.

11 Beyer CK, Albert DM. The use and fate of fascia lata and sclera in ophthalmic plastic and reconstructive surgery. The 1980 wendall hughes lecture. Ophthalmology. 1981;88: 869-86.
12 Lee J, Lee H, Chang M, Park M, Baek S. Clinical results of modified frontalis suspension technique using preserved fascia lata for congenital ptosis. J Craniofac Surg. 2016;27(5): e477-81.

13 Wilson ME, Johnson RW. Congenital ptosis. Long-term results of treatment using lyophilized fascia lata for frontalis suspensions. Ophthalmology. 1991;98(8):1234-7.

14 Whitehouse GM, Grigg JR, Martin FJ. Congenital ptosis: results of surgical management. Aust N Z J Ophthalmol. 1995;23(4):309-14.

15 Broughton WL, Matthews JG, Harris DJ. Congenital ptosis. Results of treatment using lyophilized fascia lata for frontalis suspensions. Ophthalmology. 1982;89(11):1261-6.

16 Orlando F, Weiss JS, Beyer-Machule CK, Albert DM, Shapiro A. Histopathologic condition of fascia lata implant 42 years after ptosis repair. Arch Ophthalmol. 1985;103(10): $1518-9$. 
17 Gutman FA. Sterilization with cobalt radiation of fascia lata for homotransplant. AM J Ophthalmol. 1965;59:1095-8.

18 Townsend AL, Crawford JS. Stored fascia lata for ophthalmic surgery. Can J Ophthalmol. 1969;4(4):331-8.

19 Nunnery WR. Risk of prion transmission with the use of xenografts and allografts in surgery. Ophthal Plast Reconstr Surg. 2001; 17(6):389-94.
20 Tan L, Williams M, Khan MK. Risk of transmission of bovine spongiform encephalopathy to humans in the United States: report of the council on scientific affairs. American medical association. JAMA. 1999;281: 2330-9.

21 Pruisiner SB. Genetic and infectious prion diseases. Arch Neurol. 1993;50(11):1129-53.

22 Memoranda. World Health Organization. Medicinal and other products and human and animal transmissible spongiform encephalopathies: memorandum from a WHO meeting. Bull World Health Organ. 1997; 75(6):505-13.
23 Darbord JC. Inactivation of prions in daily medical practice. Biomed Pharmacother. 1999;53(1):34-8.

24 Budka H, Aguzze A, Brown P, Brucher JM, Bugiani $\mathrm{O}$, Collinge J, et al. Tissue handling in suspected Creutzfeldt-Jacob disease (CJD) and other human spongiform encephalopathies (prion disease). Brain Pathol. 1995;5: $319-22$. 